\title{
Wechsel vom Tiers garant zum Tiers payant durch einzelne Ärztinnen und Ärzte
}

P. Meier

\begin{abstract}
Worum geht es?
In den letzten Wochen hat sich, gestützt auf Informationen aus verschiedenen Quellen, die Frage gestellt, ob einzelne Ärztinnen und Ärzte ohne weiteres vom Tiers garant in den Tiers payant wechseln können oder ob es hier gesetzliche bzw. vertragliche Vorgaben gibt, die eingehalten werden müssen.
\end{abstract}

\section{Regelung nach KVG}

Es gilt der Grundsatz, dass die Versicherten den Leistungserbringern die Vergütung der Leistung schulden, sofern die Versicherer und die Leistungserbringer nichts anderes vereinbart haben. Nach Gesetz gilt grundsätzlich der Tiers garant.

\section{Was gilt gemäss den mit santésuisse abgeschlossenen Verträgen?}

Es sind zwei Vertragswerke von Bedeutung: Das eine ist der Rahmenvertrag TARMED, der von der FMH mit santésuisse abgeschlossen und vom Bundesrat genehmigt worden ist. In diesem Vertrag ist der Grundsatz festgehalten, dass Schuldner der Vergütung von ärztlichen Leistungen im Rahmen des KVG der Versicherte ist (Tiers garant). Ärzte und Versicherer können aber auch das System des Tiers payant vereinbaren (Art. 11 $\mathrm{RV})$. Hier ist also offengelassen, wer welches System wählt.

\section{Kantonale Anschlussverträge zum Rahmenvertrag TARMED}

Die kantonalen Anschlussverträge wurden von jedem Kanton oder von jeder Region mit santésuisse abgeschlossen. In diesen Verträgen ist nun klar geregelt, nach welchem System (TG, TP) abgerechnet werden soll. So steht z. B. in den Verträgen AG und SO in Art. 11 AV ganz klar: «Schuldner gegenüber dem Leistungserbringer im Rahmen des KVG ist der Versicherte (System des Tiers garant). Der Arzt schickt die Rechnung dem Versicherten.»
Das System Tiers payant ist einzig in folgenden Kantonen im Anschlussvertrag vereinbart worden: GL, GR, SG, SZ und UR.

So steht z.B. im Bündner Anschlussvertrag folgendes: «Schuldner gegenüber dem Leistungserbringer ist der Versicherer (System des Tiers payant).»

\section{Welches sind die Vertragspartner der Anschlussverträge?}

Die Verträge wurden bekanntlich von den kantonalen Gesellschaften, eventuell regional (z.B. Innerschweiz), abgeschlossen, aber - und dies ist wichtig - sämtliche Ärztinnen und Ärzte sind diesen Verträgen automatisch beigetreten, es sei denn, einzelne Ärztinnen und Ärzte haben ihren Gesellschaften mitgeteilt, dass sie den Anschlussverträgen nicht beitreten. Die Verträge sind von den Kantonsregierungen genehmigt worden und demnach verbindlich.

\section{Pacta sunt servanda}

Die Anschlussverträge, die für alle beigetretenen Ärztinnen und Ärzte verbindlich sind, können gekündigt werden, und zwar innert folgender Fristen: Rahmen- und Anschlussvertrag können erstmals auf das Ende des 18. Monates nach Einführungsdatum, d.h. erstmals per 30. Juni 2005, gekündigt werden. Die Kündigungsfrist beträgt 6 Monate (Art. 18 RV). Dies ist der Grundsatz.

Wichtig ist aber Art. 4 des Anschlussvertrages. Dort ist der Rücktritt vom Vertrag für einzelne Ärzte geregelt. Diese Bestimmung lautet z.B. im Anschlussvertrag AG und SO wie folgt: «Einzelne Ärzte und Versicherer können unter Einhaltung einer Frist von 6 Monaten jeweils per 30.06. und per 31.12. von diesem Vertrag zurücktreten. Der Rücktritt kann frühestens nach Ablauf der Kostenneutralitätsphase erfolgen.»

Wann endet die Kostenneutralitätsphase? Gemäss Art. 18 Kostenneutralitätskonzept erstreckt sich die Kostenneutralitätsphase vom April des Einführungsjahres bis zum April des Folgejahres, also bis zum April 2005. 


\footnotetext{
* Der Patient ist auch im Tiers payant Herr seiner Rechnungsdaten! Er kann formlos auf Kassenleistungen verzichten. Dies muss er aber vor dem Versand der Rechnung an die Krankenversicherung wissen. Nur dann kann er entscheiden, ob die Rechnung in die Hand der Kasse gelangen soll oder ob er «faute de mieux» die Behandlung selbst bezahlt. Das ist im Tiers payant faktisch ausgeschlossen und schadet damit potentiell dem Arzt-PatientenVertrauensverhältnis. Der Patient kann überrumpelt werden, wenn die Kasse zuerst über die Rechnungsdetails verfügt.
}

\section{Zusammenfassung}

- Der Wechsel einzelner Ärztinnen und Ärzte in den Tiers payant, der auf Anraten von Abrechnungsstellen oder Intermediären erfolgte, ist vertragswidrig.

- Durch ihren Systemwechsel gefährden sie die Kostenneutralität (es kommt zu Volumenverschiebungen).

- In den meisten Fällen wurden die Patienten über den Wechsel nicht informiert. Gemäss Vorgaben des Datenschutzes müssen die Patienten einem direkten Versand der Rechnungen an die Versicherer zustimmen.*

- Ein Wechsel vom Tiers garant zum Tiers payant kommt einer Vertragsänderung (für den einzelnen Arzt) gleich. Der Vertrag müsste mit den Versicherern speziell ver- einbart werden (mit santésuisse oder jedem einzelnen Versicherer), und er bedarf auch der Zustimmung des zuständigen Regierungsrates.

\section{Empfehlungen}

1. Verzichten Sie während der Dauer der Kostenneutralitätsphase auf einen Wechsel vom Tiers garant zum Tiers payant.

2. Falls Sie dies schon gemacht haben, nehmen Sie mit Ihrer Kantonalgesellschaft Kontakt auf.

3. Unterbreiten Sie Aufforderungen von Dritten, in den Tiers payant zu wechseln, Ihren Kantonalgesellschaften zur Stellungnahme.

\title{
Certains médecins passent du système du «tiers garant» à celui du «tiers payant»
}

\author{
P. Meier
}

Correspondance:

Peter Meier

Avocat et notaire /

Conseiller juridique du G7

Schmiedengasse 33

CH-5012 Schönenwerd

Tél. 0628494200

E-mail: aeg.so@bluewin.ch

\section{De quoi s'agit-il?}

Ces dernières semaines, différentes sources d'information ont révélé qu'on se posait actuellement la question de savoir si des médecins pouvaient, à titre individuel, passer sans autre du système du tiers garant à celui du tiers payant ou s'il existait à ce propos des dispositions légales ou contractuelles à respecter.

\section{Réglementation selon la LAMal}

Pour autant qu'assureurs et médecins n'aient pas convenu autre chose, le principe prévaut selon lequel les assurés sont les répondants des coûts face aux fournisseurs de prestations et qu'ils doivent, à ce titre, payer leurs prestations. En vertu de la LAMal, c'est donc le principe du tiers garant qui est applicable.

\section{Quels sont les principes applicables en vertu des conventions passées avec santésuisse?}

Deux sortes de conventions sont importantes. Il $\mathrm{y}$ a tout d'abord la convention-cadre TARMED conclue entre la FMH et santésuisse et approuvée par le Conseil fédéral. Cette convention précise que l'assuré est le débiteur du remboursement des prestations médicales fournies dans le cadre de la LAMal (tiers garant). Médecins et assureurs peuvent cependant aussi convenir du système du tiers payant (art. 11 de la convention-cadre). Le choix du système reste ouvert. 


\section{Conventions cantonales d'adhésion à la convention-cadre TARMED}

Il y a ensuite des conventions cantonales d'adhésion, qui ont été conclues par chaque canton ou chaque région avec santésuisse. Dans ces conventions, le système de facturation à appliquer (tiers garant, tiers payant) est clairement indiqué. Les cantons d'Argovie et de Soleure prévoient par exemple à l'article 11 que les débiteurs des fournisseurs de prestations sont, dans le cadre de la LAMal, les assurés (système du tiers garant). Le médecin envoie donc sa note d'honoraires à l'assuré.

Le système du tiers payant (les débiteurs des fournisseurs de prestations sont les assureurs) figure dans les conventions cantonales d'adhésion uniquement pour les cantons suivants: Glaris, Grisons, St-Gall, Schwytz et Uri.

\section{Quels sont les partenaires contractuels des conventions cantonales d'adhésion?}

Ces conventions ont été conclues par les sociétés cantonales de médecine, éventuellement aussi sur le plan régional (p.ex. Suisse centrale) mais, et c'est important, tous les médecins y sont automatiquement affiliés, à moins que certains d'entre eux n'aient communiqué à leur société qu'ils renonçaient à y adhérer. Les conventions ayant été approuvées par les gouvernements cantonaux, elles revêtent un caractère obligatoire.

\section{Pacta sunt servanda}

*ême dans le système du tiers payant, le patient reste le maitre des données de ses factures! Il peut sans autre renoncer à des prestations de la caisse-maladie mais doit savoir à l'avance que sa facture sera transmise à l'assurance. A ce moment seulement, il pourra décider s'il approuve cette manière de faire ou, faute de mieux, s'il préfère payer lui-même le traitement. Dans le système du tiers payant, ce libre choix est pratiquement exclu, ce qui nuit au rapport de confiance entre le patient et le médecin. Le patient peut être pris au dépourvu si la caisse dispose de données détaillées avant lui.
Les conventions cantonales d'adhésion, dont l'application est obligatoire pour tous les médecins affiliés, peuvent être résiliées moyennant les délais suivants:

La convention-cadre et les conventions d'adhésion peuvent être résiliées la première fois pour la fin du $18^{\mathrm{e}}$ mois qui suit la date de leur introduction, c'est-à-dire la première fois pour le 30 juin 2005. Le délai de résiliation est de 6 mois (art. 18 de la convention-cadre). C'est donc la norme à respecter.

Mais l'article 4 de la convention d'adhésion est important car il règle la démission des méde- cins à titre individuel. La convention d'adhésion du canton d'Argovie et de Soleure stipule, p.ex. que les médecins et assureurs peuvent, moyennant un délai de 6 mois, démissionner personnellement pour le 30 juin et le 31 décembre. Le retrait de la convention peut avoir lieu au plus tôt à l'expiration de la phase de neutralité des coûts.

Quand la phase de neutralité des coûts prend-elle fin? Selon l'article 18 du concept de la neutralité des coûts, cette phase s'étend du mois d'avril de l'année d'introduction au mois d'avril de l'année suivante, donc jusqu'en avril 2005.

\section{Récapitulation}

- Le passage de certains médecins au système du tiers payant sur le conseil d'organes de facturation ou d'intermédiaires, contrevient à la convention.

- Par ce changement, ils mettent en danger la neutralité des coûts (des décalages se produisent dans les volumes de prestations).

- Dans la plupart des cas, les patients n'ont pas été informés de ce changement. Or, selon les directives de la protection des données, les patients doivent approuver l'envoi direct des factures aux assureurs.*

- Le passage du système du tiers garant à celui du tiers payant équivaut à une modification contractuelle (pour le médecin). La clause contractuelle y afférente doit être spécialement convenue avec les assureurs (avec santésuisse ou chaque assureur en particulier); elle nécessite aussi l'approbation du gouvernement cantonal concerné.

\section{Recommandations}

1. Nous vous recommandons de renoncer à passer du système du tiers garant à celui du tiers payant pendant la durée de la neutralité des coûts.

2. Si vous l'avez déjà fait, veuillez prendre contact avec votre société cantonale.

3. Soumettez à votre société cantonale pour prise de position les invitations à passer dans le système du tiers payant. 